\title{
MUJERES DEL HOTEL TASELOTZIN, EMPODERADAS Y EMPRENDEDORAS
}

Por. Dra. Ivonne Tapia Villa Gómez, Dr. Héctor Manuel Villanueva Lendechy Universidad Ibero de Puebla. México

Ivonne.tapia@iberopuebla.mx, hector.villanueva@iberopuebla.mx

\section{RESUMEN}

El emprendimiento es un concepto que aún está en construcción y al cual, aún se le deben de abonar varias investigaciones empíricas. Sin embargo, varios autores convergen que el fin primordial es el beneficio social por encima del interés económico y financiero de quien emprende. Cuando este tipo de emprendimientos, los llevan a cabo mujeres que buscan llevar recursos económicos a sus hogares y que se asocian con otras mujeres que tienen necesidades similares, utilizando recursos del campo o aquellos que de manera tradicional se han venido elaborando procurando la sustentabilidad y la sostenibilidad. Se identifica la relación entre el empoderamiento de estas mujeres rurales con el emprendimiento que llevan a cabo. Esta investigación fue de tipo cualitativa y utilizó el paradigma interpretativista. El método utilizado fue inductivo y las técnicas utilizadas fueron la documental y la entrevista de panel. El instrumento fue el cuestionario semiestructurado y el procedimiento de muestreo fue no probabilístico a juicio de los investigadores con base en su experiencia. Sus variables fueron el empoderamiento de la mujer rural con las categorías: negocio propio, cultura, capacitación. Las informantes fueron cuatro mujeres que forman parte de la organización del Hotel Taselotzin, que se encuentra ubicado en el municipio de Cuetzalan del Estado de Puebla. Para procesar y analizar los datos se utilizó el software ATLAS-ti.

Palabras clave: Emprendimiento social, empoderamiento, impacto social, sostenibilidad.

\section{INTRODUCCIÓN}

El capitalismo neoliberal ha sido un sistema que ha polarizado la distribución de la riqueza, es decir, solo unos cuantos tienen un gran poder económico y político. En cambio, la mayor parte de la población vive en condiciones de pobreza. Otra consecuencia es el deterioro ambiental del planeta, que en consecuencia ha provocado la desaparición de muchas especies animales y vegetales. Aunado a lo anterior, en muchas empresas las condiciones laborales son contrarias al trabajo decente, es decir que, no existe respeto por la dignidad humana y la retribución económica no es justa porque no cubre las necesidades más apremiantes. En relación con ello, se considera que los emprendimientos sociales son un contrapeso de las empresas capitalistas tradicionales que promueven el desarrollo sostenible, entendiéndose este como aquel que asegura los recursos para que las generaciones venideras puedan hacer uso de ellos.

\section{EL EMPRENDIMIENTO SOCIAL.}

Los emprendimientos sociales, como estructuras productivas, tienen una impronta de beneficio social, y en este sentido, se puede afirmar que son formas alternativas de empresa dentro de un capitalismo neoliberal que cobija a las organizaciones orientadas al lucro, a la acumulación de capital y a enriquecer a unos cuantos originando la polarización entre la ciudad y el campo. En contrasentido, en los emprendimientos sociales se fomenta el trabajo cooperativo y la toma de decisiones de manera consensuada o democrática, los socios son a la vez los trabajadores, y estos determinan cómo y en qué se aplicarán los beneficios y se distribuirán las ganancias. Además, utilizan técnicas administrativas que contribuyen a la sostenibilidad y la sustentabilidad del emprendimiento.

Las características del emprendimiento social, después de la revisión de algunos autores (Palacios, 2010; Kliksberg, 2011; Alonso, González y Nieto, 2015; Rodríguez y Flores, 2016) son las siguientes: 
1. El germen embrionario del bien social; 2 . Un cambio social sostenido, lo que implica: a) La sostenibilidad de la organización (independencia financiera y rentabilidad) y b) La consecución del cambio social de manera continua; 3 . El mejoramiento de las condiciones de por lo menos un grupo; 4 . El uso de métodos y técnicas de gestión; 5 . La posibilidad de nuevos emprendimientos, eliminando con ello la co-dependencia; 6. Se considera que el emprendimiento social se gesta como parte de una dinámica en la cual se genera y socializa el conocimiento, los agentes del cambio y además se fortalece el capital social de sus miembros.

\section{EMPODERAMIENTO RURAL FEMENINO EN MÉXICO}

Las mujeres rurales (en su anhelo por lograr satisfacer las necesidades básicas de su hogar, su rol económico y político, acceso a la tierra y la toma de decisiones) han generado el fenómeno de "feminización del campo o de la agricultura" (CEDRSSA, 2014; Garay, 2015; López Guerra, et al, 2017). Es por ello que más mujeres emprenden un negocio propio con los recursos del agro que disponen o que se les otorgan por medio de las remesas, iniciando así un proceso de empoderamiento personal, económico y político-social (Vázquez, Mortera, Rodríguez, Martínez y Velázquez, 2013; Soler, et al 2014).

El estudio del empoderamiento de la mujer se gesta principalmente en América Latina a partir de movimientos colectivos de mujeres en defensa de sus derechos. De ahí que no solo se debe hablar solo del empoderamiento económico de la mujer, sino del empoderamiento que nace a partir de la colectividad femenina que defiende causas políticas, influyendo en la transformación de instituciones que por antonomasia ha sido dirigida por hombres.

De manera específica, el empoderamiento rural femenino se relaciona con variables como el grado académico, la inversión en negocios, dedicarse al comercio, alto grado de disposición para el trabajo comunitario, satisfacción al ejercer el trabajo remunerado, entre otras (Vázquez et al, 2013).
Sin embargo, los hallazgos de investigaciones de campo de algunos de los autores de esta investigación, demostraron que los emprendimientos de mujeres rurales indígenas en comunidades del Estado de Veracruz, se llevan a cabo de común acuerdo con su pareja masculina, lo cual contraviene algunas otras investigaciones, cuya condición sine qua non para la feminización del campo es la carencia de hombres por migración, muerte o separación

\section{METODOLOGÍA}

La investigación se llevó a cabo el 29 de junio de 2018 en el Hotel Taselotzin en el municipio de Cuetzalan del estado de Puebla. Se utilizó el paradigma cualitativo, de corte interpretativista. Por lo tanto, se recurrió al método inductivo, las técnicas que se utilizaron fueron la documental y la entrevista de panel o grupo de discusión in situ, el instrumento fue el cuestionario semi-estructurado y el procedimiento de muestreo fue no probabilístico a juicio de los investigadores con base en su experiencia, mediante un consenso de los mismos. Los informantes fueron 4 mujeres que forman parte de la empresa de responsabilidad social del hotel referido, las entrevistadoras fueron investigadoras ya que se consideró pertinente que no fueran hombres, dada la perspectiva de género de las administradoras del hotel. Para procesar los datos, se utilizó el software ATLAS-ti y el procedimiento fue el siguiente: antes de realizar la investigación de campo se determinaron las categorías con base en la revisión literaria y la experiencia de los investigadores. Posteriormente, se llevó a cabo una lectura analítica de la transcripción de la entrevista, se codificó, identificando las subcategorías. A continuación, se ingresaron los datos en el software para su análisis, a partir de esto se generó la tabla de co-ocurrencia y las redes semánticas. Para fines de esta etapa de presentación de resultados, solo se llevaron a cabo las redes de las categorías de negocio propio, cultura y capacitación. El objetivo de la investigación fue identificar como se relacional el empoderamiento indígena femenino y el emprendimiento social en el hotel Taselotzin.
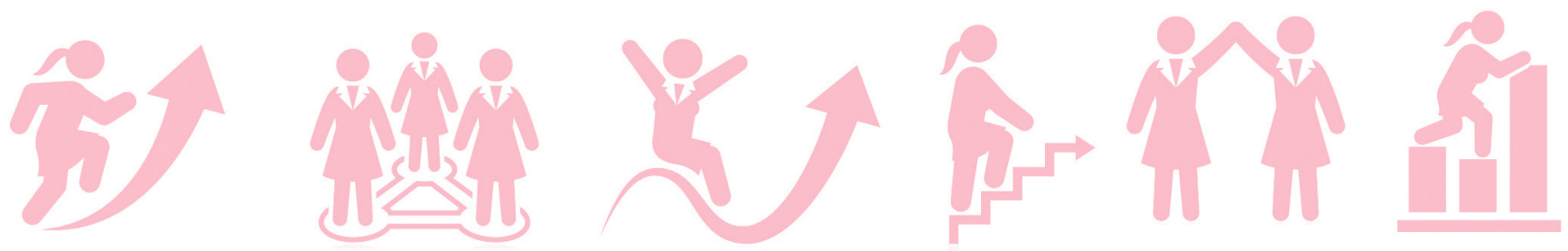


\section{RESULTADOS}

El hotel Taselotzin está ubicado en la Sierra Norte de Puebla, de manera particular en Cuetzalan, se empezó a construir en el año de 1996 y se inauguró en 1997 con servicio de hospedaje y alimentos por la organización Masehual Siuamej Mosenyolchicauani, un nombre náhuatl que en español significa: mujeres indígenas que se apoyan. Esta empresa es consecuencia de una serie de actividades económicas que algunas mujeres de la comunidad llevaron a cabo, con la finalidad de contribuir a los ingresos económicos del hogar cuando estos escasearon como consecuencia del cambio climático que provocó un aumento en las precipitaciones pluviales y como consecuencia los campesinos dejaron de sembrar y comercializar sus cosechas, lo cual disminuyó los ingresos hacia los hogares (Masehual, 2016).
Los servicios que se ofrecen en el hotel son: habitaciones (diez), albergues (dos), restaurante, servicio de masaje relajante, masaje tradicional, temazcal tradicional (dos), limpia curativa, recorridos guiados y talleres de idioma náhuatl y demostración a grupos de: taller de cintura, pláticas de herbolaria y sobre la historia de la organización. Además, algunas de las acciones sustentables son: fosa séptica, trampas de aceite y un área de reserva ecológica.

Después de analizar los datos relacionados con las categorías de emprendimiento social, se generó la red semántica de la categoría de negocio propio, la cual se presenta a continuación con sus subcategorías:

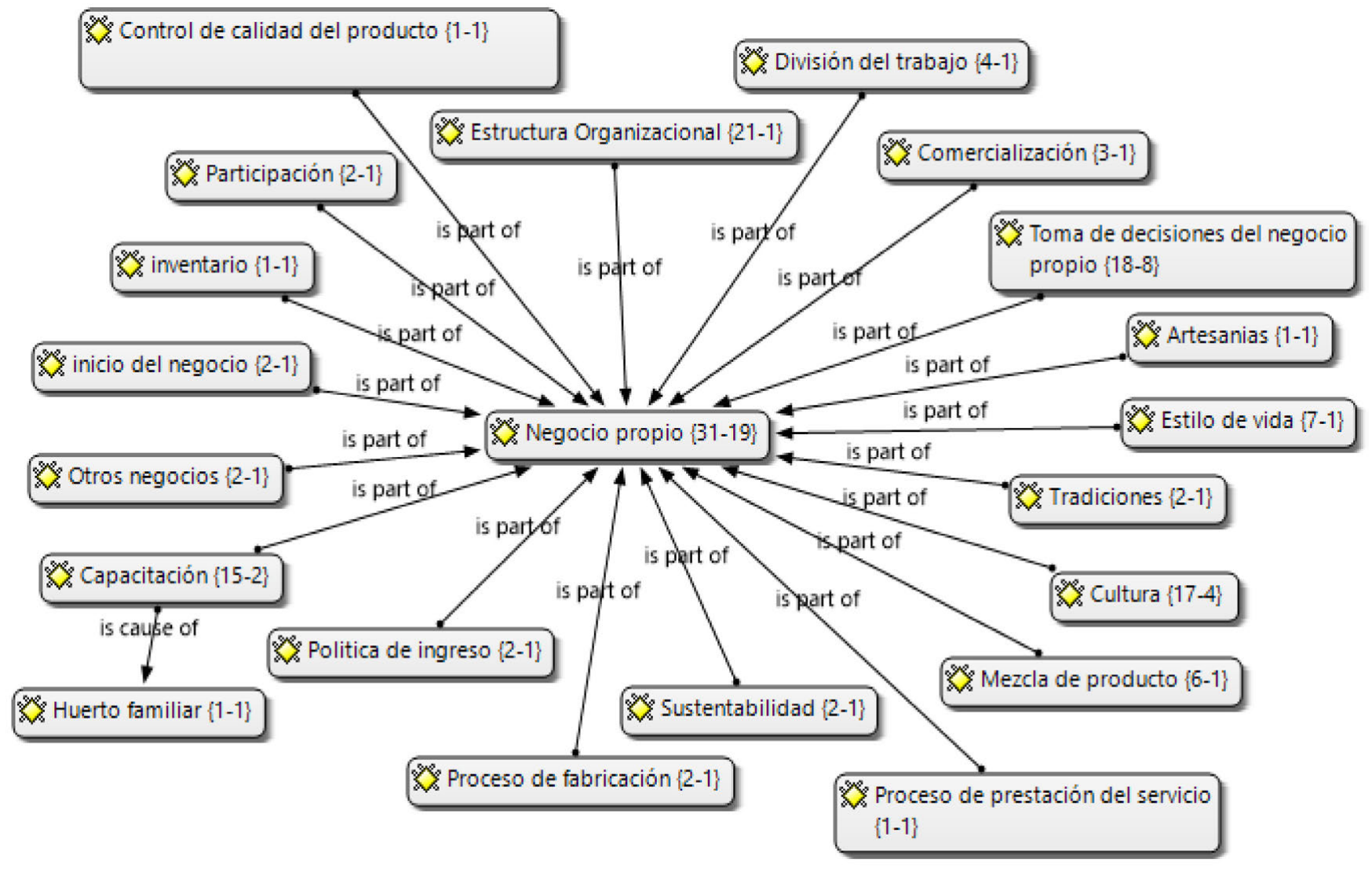

Figura 1. Red semántica de la categoría de negocio propio.

Fuente: elaboración propia.
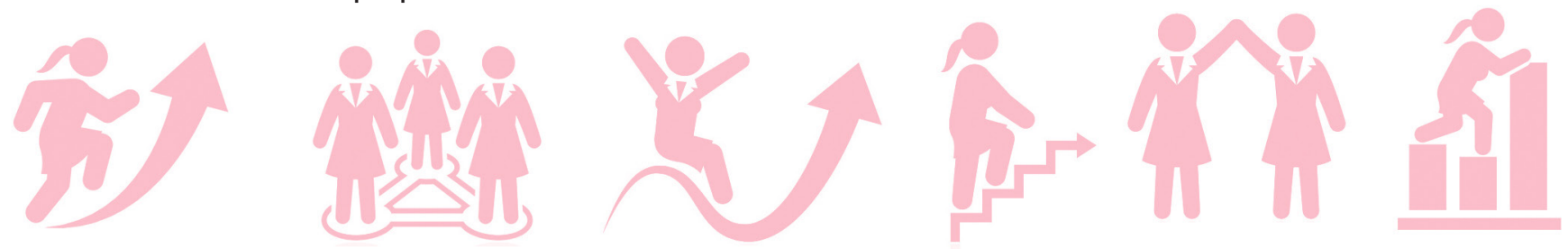
Análisis de co-ocurrencias.

Además de diseñar la red semántica de negocio propio, se desarrolló mediante el software ATLAS-ti, un análisis entre sus categorías y subcategorías para determinar la co-ocurerencia entre ellas, es decir, las cantidades que resultan del cómputo del número de veces que dos o más unidades léxicas están presentes contemporáneamente en los mismos contextos elementales o sea en la entrevista.

Con base en lo anterior, se identificó que la relación más fuerte de esta categoría fue con la subcategoría de estructura organizacional $(0.63$, en una escala de 0 a 1) porque se observaron 21 comentarios de ésta respecto a las del negocio propio. La estructura organizacional se refirió al proceso de ingreso a la organización estudiada, las responsabilidades y funciones del puesto de las mujeres que trabajan en el hotel Taselotzin, las actividades que realizan los hombres y el procedimiento de sucesión para administrar el hotel.

A continuación, se presentan algunos fragmentos de la transcripción de la subcategoría de estructura organizacional:

Ivonne: para entrar a la organización comentaba que ¿no hay un requisito especial?

Daniela: antes no pero ahorita ya, sí.

Ivonne: ¿Qué requisitos son?

Daniela: primero, primero debe...(inaudible) debe presentarse en su comunidad con el grupo, ir a ir a hablar, si aceptamos o no aceptamos, entonces de ahí ya le decimos que sí. Ya cuando hace la asamblea aquí, ya se viene a presentar y entrega su acta de nacimiento, su copia de credencial, su CURP, ya lo entregan aquí en la organización, entonces ya se registran...(inaudible).

Ivonne: ¿cuál es la relación que tienen con el hotel? Porque aquí venden sus prendas.

Todas: (inaudible y risas).

Juanita: pues, antes que nada, buenos días, mi nombre Juana María Nicolás Chepe, soy la responsable del Hotel Taselotzin, nuestra organización lleva 33 años que estamos trabajando como organización Masehual Siuamej, nuestras compañeras son ellas nuestras dirigentas, les están preguntando pero dicen que les falta para contestarlas. Ellas son las directivas de cada comunidad, son del Consejo de Mujeres a nivel regional, este...este somos 6 comunidades que estamos trabajando, estamos este (inaudible) San Miguel Zinacápan (inaudible) Tepextla, de esas seis comunidades tienen sus directivas, tienen su consejo a nivel regional y tienen sus directivas de cada comunidad.

En contraste, las relaciones más débiles (0.30) fueron con las subcategorías: artesanías, control de calidad del producto, estilo de vida, huerto familiar, inicio del negocio, inventario, participación y proceso de prestación de servicios, sustentabilidad y tradiciones porque la frecuencia del comentario fue de solo una vez. En el mismo orden de ideas, pero respecto a la categoría de rango de toma de decisiones del negocio propio, la red semántica es la siguiente:
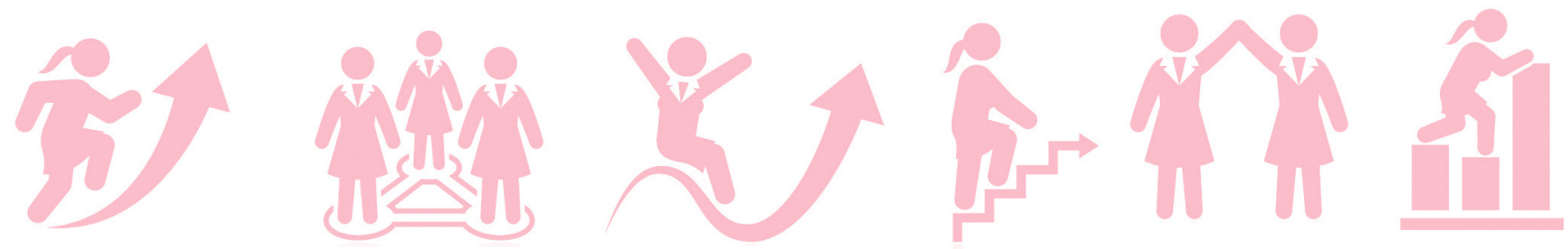
En este mismo sentido, también se generó la red semántica de la categoría de cultura y sus subcategorías, la cual es la siguiente:

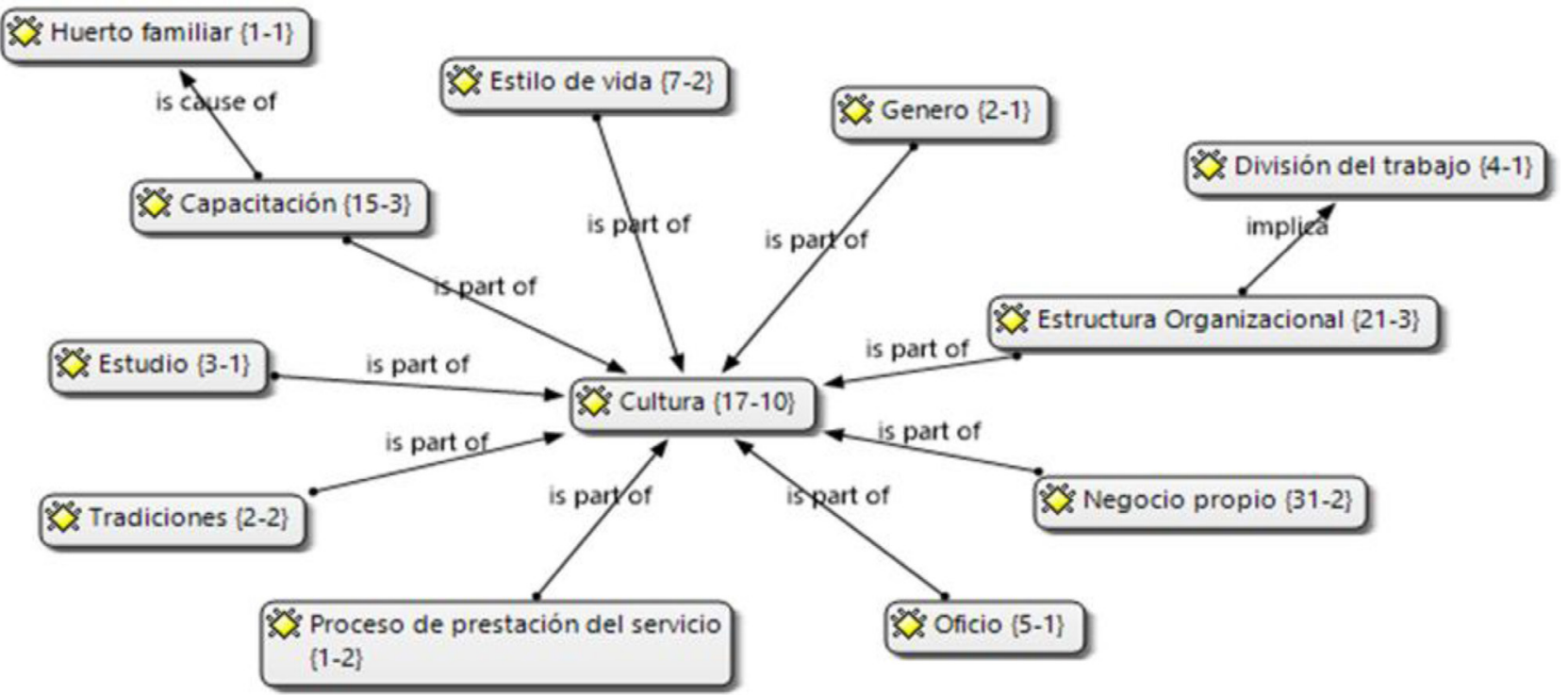

Figura 2. Red semántica de la categoría de cultura. Fuente: elaboración propia.

\section{Análisis de co-ocurrencias.}

Además de diseñar la red semántica que involucra a la cultura como categoría, también se desarrolló mediante el software ATLAS-ti, un análisis para determinar su co-ocurrencia con sus subcategorías. La co-ocurrencia se relaciona con las cantidades que resultan del cómputo del número de veces que dos o más unidades léxicas están presentes contemporáneamente en los mismos contextos elementales 0 sea en la entrevista. La relación más fuerte fue con la subcategoría de estilo de vida (0.41), es decir que los comentarios relacionados entre cultura y la subcategoría denominada estilo de vida fueron los que más frecuentemente se mencionaron en la entrevista, en contraposición, la relación más débil fue con la subcategoría de estructura organizacional (0.03) lo que significa que los comentarios que relacionan a ambos elementos fueron los menos frecuentes.

Con relación a lo anterior, para llevar a cabo otro tipo de análisis, se presentan los fragmentos de las transcripciones relacionados con la categoría de cultura fueron los siguientes:

Elena: entre ustedes en la comunidad, ¿son parientas? De qué hija, hermana, tía o ¿puede entrar cualquier mujer?
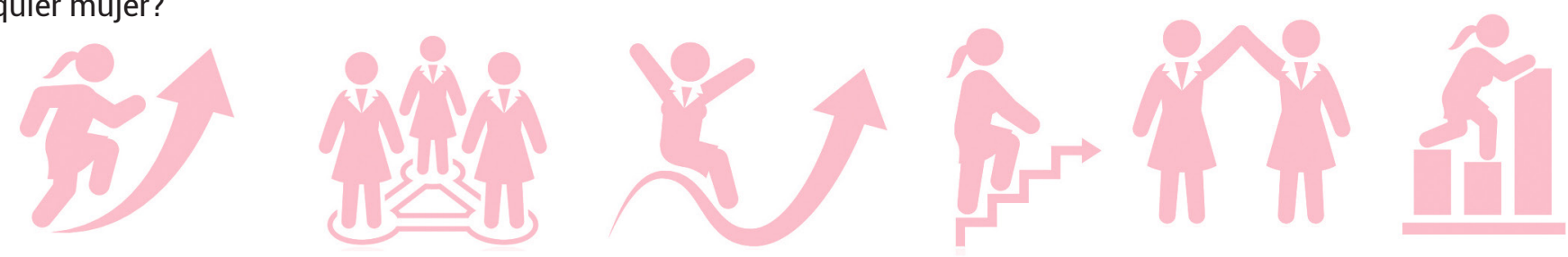

Petra: no, este, invitamos a cualquier persona.

Elena: porque estamos hablando que en la comunidad son lugares pequeños ¿verdad? Es pequeño.

Daniela: pues no tanto, ya hay más gente pues...(inaudible) antes entraban más, pero (inaudible) ya no viven las señoras (inaudible)...tres personas.

Elena: ¿ya murieron?

Daniela: si, ya. Ya las...(inaudible) ahorita ya no quieren entrar, estudian y ya saliendo ya se van...(inaudible).

Elena: entonces, más bien las jovencitas ya no...

Daniela: ya no quieren, ya no quieren coser, más bien... (inaudible).

Elena: y si van a la escuela ¿viene acá?

Daniela: pues otras...(inaudible) van a otro lado...(inaudible).

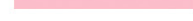


Ivonne: ¿cómo van a hacer para que estas nuevas generaciones están tomando mejor la decisión de mejor irse a estudiar? ¿Cómo le van a hacer para preservar esta parte de los bordados, de los textiles, que no se pierda la tradición que ha sido parte fundamental de su comunidad? ¿Cómo le han hecho en cierto modo para preservar esta tradición?

Daniela: pues una ya quería entrar (inaudible) como otras que ya no quieren estudiar, quieren entrar, quieren aprender los bordados, pues ya (inaudible) unas van y otras se quedan...sí.

Ivonne: ¿ustedes les enseñan?
Daniela: sí, nosotros les enseñamos. Ahí en la familia uno, hay muchas familias que pueden hacer bordado allá, pero no todos quieren entrar en la organización, están en la casa (inaudible).

El análisis de los fragmentos de la transcripción, develan que la fabricación de las artesanías, es parte de la cultura que se ha heredado de generación en generación. Sin embargo, las generaciones actuales de mujeres tienen menos interés por aprender el tejido de cintura, lo cual, plantea las preguntas ¿quiénes reproducirán esta forma de tejer? Y ¿está en riesgo de perderse esta técnica de tejido?

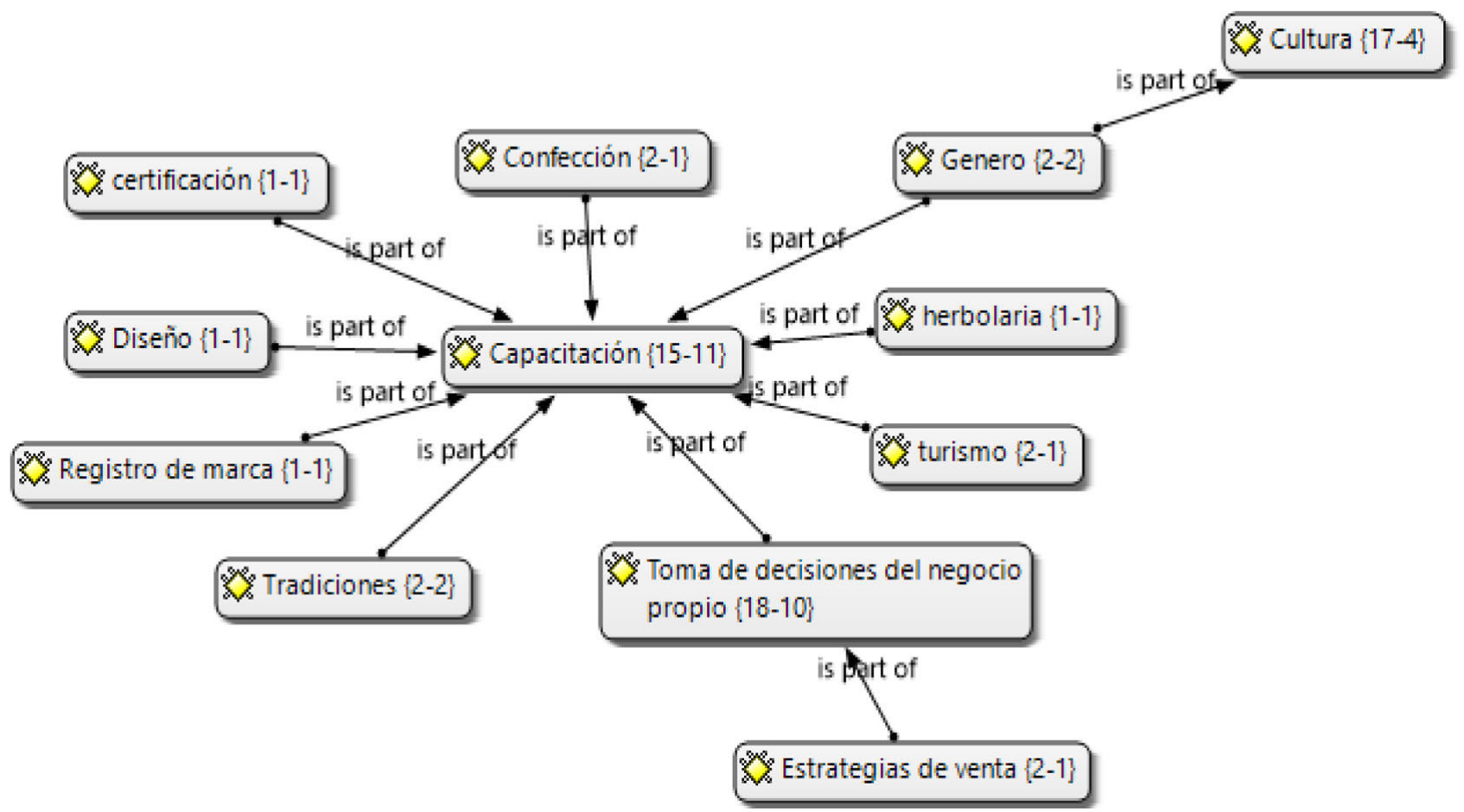

Figura 3. Red semántica de la categoría capacitación.

Fuente: elaboración propia.

Tabla de co-ocurrencia.

Las relaciones más fuertes de la categoría de capacitación se presentaron con las subcategorías de a) confección de prendas; b) temas de género y; c) turismo (co-ocurrencia del 0.13 , en una escala de 0 a 1 , en los tres casos). Es decir, que en la entrevista las mujeres mencionaron más veces las subcategorías antes referidas cuando se trató el tema de capacita- ción. La relación con menos fuerza fue con la subcategoría de cultura (0.03), es decir que en la entrevista se mencionó menos veces la subcategoría de cultura al referirse al tema de capacitación. La relación entre capacitación y cultura se generó porque se observó que las nuevas generaciones muestran poco interés en continuar con las tradiciones, por ejemplo, el tejido de cintura y bordado.
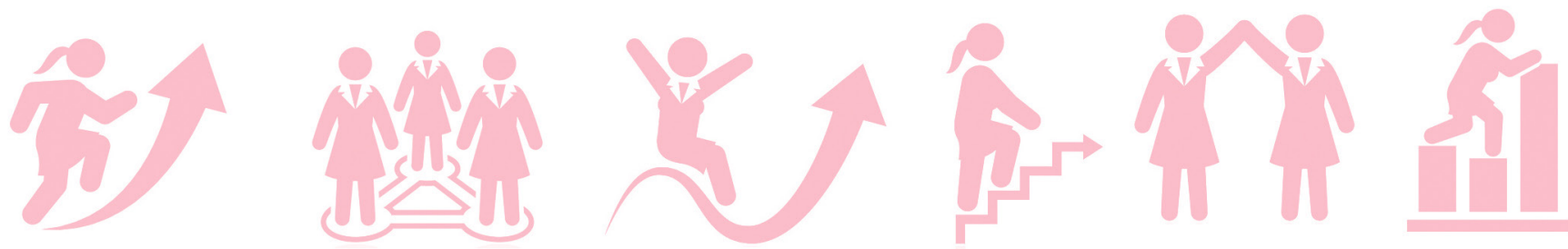
Algunos fragmentos de las transcripciones relacionados con la subcategoría de capacitación fueron:

Ivonne: ¿cursos de qué? ¿de bordado?

Yolanda: o sea que nosotros hacemos el bordado y solo nos enseñan cómo armarlo, cómo hacer una blusa para que tenga buenas terminaciones para que no se vea así, así tan asobronada, ora si, para que se vea bien...

Ivonne: ¿vienen de algún lugar estas personas a darles los cursos?

Yolanda: sí, sí.

Ivonne: ¿de qué lugar vienen?

Yolanda: pues vienen de México.

Ivonne: ¿de alguna organización en especial?

Yolanda: yo creo que si porque son de Corazón Verde.

Ivonne: ¿esto es algo habitual? ¿Ustedes ya tienen pactado que vengan estas personas, y en estas fechas que dicen de diciembre, mayo? ¿Ya saben que van a venir ellos (inaudible)?

Yolanda: la de corazón verde sí, es lo que estamos platicando, que si vemos que ...(inaudible) le vamos a seguir...(inaudible)...

Ivonne: $Y$ ya en otro sentido, ¿usted cómo se siente al poder tener esta capacidad de poder genera sus propios recursos sin necesidad de que haya un hombre que la apoye y hay otra manera y ustedes están tomando sus decisiones, son autosuficientes y como inspiran también a otras mujeres?

Juanita: bueno este, yo pienso este, nosotros pensamos que sí se puede hacer el trabajo porque este como decían la compañera Petra...porque este como les decía pues tomamos decisiones nosotros como mujeres indígenas, porque de hecho hemos tomado capacitaciones. Las compañeras ellas saben más cuales son los derechos de las mujeres indígenas, porque Doña Dany, ella está con las compañeras del cambio ellas están tomando cursos de capacitación sobre la violencia...

Juanita Chepe: ...Al inicio, pues difícil fue porque sabíamos que somos de las comunidades que es diferente que unos manejamos que no lo conocemos, entonces ahí fuimos aprendiendo cursos, capacitaciones, en parte del turismo también ellos nos han dado capacitaciones por parte de SECTUR (Secretaría de Turismo) de Puebla.

Ellos nos han invitado a capacitaciones para que participamos todo el equipo de trabajadores y también este hemos tomado documentos descriptivos hemos metido a curso de capacitación y también este pues así hemos aprendiendo todos...

Fabiola: ¿Cuándo empezaron este proyecto del hotel, tenían ya algún conocimiento sobre la administración y la puesta del proyecto o como iniciaron?

Juanita: ...mmm, pues de hecho como les decía, la organización lleva 33 años, las compañeras del consejo participaban de las administraciones y llevaban las reuniones, y pues llevaban el control de las artesanías. De hecho, con eso empezamos nuestras artesanías, empezamos capacitándonos de administración...y pues ahí fuimos aprendiendo de la administración, como es registro de las artesanías, porque las compañeras que traían se tenían que registrarse cada su prenda y ponerle su precio, y ahí fuimos aprendiendo...

Con base en lo anterior, algunas de las capacitaciones que recibieron las mujeres fueron en administración de empresas, registro de marca, diseño de bordados, corte y confección de camisas, comercialización de productos, aspectos relacionados con el distintivo $M$, además en temas de derechos de las mujeres indígenas y violencia hacia las mismas. Algunas de las organizaciones e instituciones que les han impartido los cursos son Corazón Verde, Universidad Autónoma Metropolitana (UAM) de Xochimilco, la Universidad Iberoamericana Puebla y de México, Secretaría de Agricultura, Ganadería, Desarrollo Rural, Pesca y Alimentación (SAGARPA) y Secretaría de Turismo (SECTUR).
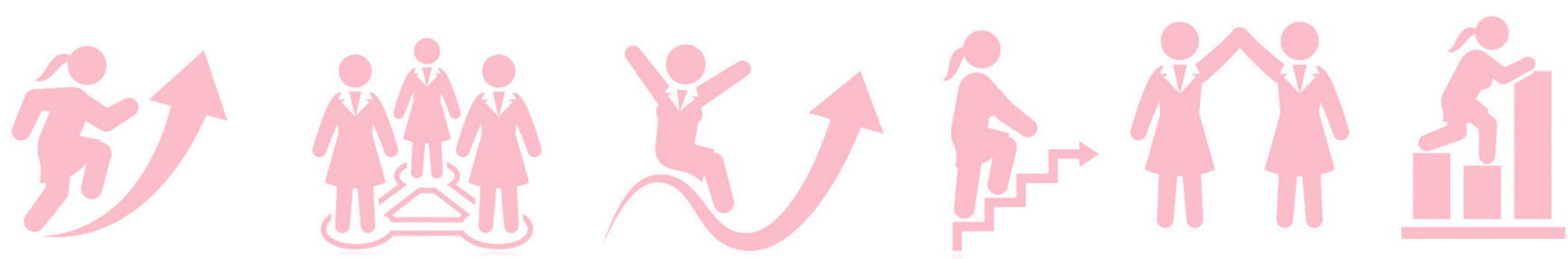


\section{CONCLUSIONES}

Se considera que existe una relación entre el empoderamiento de la mujer rural indígena y el empoderamiento rural femenino en el Hotel Taselotzin. Esta relación se estudió con las categorías de la variable denominada empoderamiento de la mujer rural indígena: negocio propio, cultura y capacitación. A partir de lo anterior, se concluye que el negocio propio se relaciona con la cultura porque con base en esta, las mujeres han determinado los tipos de negocios y los productos que venden en ellos, por ejemplo la producción en telares de cintura, los productos de medicina tradicional, el servicio de temazcal, etc., además de sus acciones orientadas hacia la sustentabilidad (categoría) como la siembra y preservación de plantas medicinales, separación de desechos en orgánicos e inorgánicos, la fosa séptica y la adquisición de áreas verdes para destinarlas a reserva territorial.

Este emprendimiento social también se caracteriza, porque se valora la cultura de las comunidades, en este sentido, los emprendimientos de las mujeres en cuestión se relacionan con su bagaje cultural y se materializan con la fabricación de las artesanías tales como las camisas y los bordados en telar de cintura, los productos relacionados con la herbolaria, la medi- cina tradicional y servicios como el temazcal, incluso el cuidado al medio ambiente es parte de su cosmovisión. Las administradoras del hotel privilegian los negocios con proveedores que pertenecen a las comunidades de la Sierra Norte de Puebla, incluso algunos son sus vecinos, por lo cual se puede vislumbrar una red de comercialización basada en la confianza y en el interés por preservar las tradiciones y costumbres de las comunidades involucradas.

Las mujeres rurales indígenas que emprenden negocios comunitarios generalmente no tienen la capacitación administrativa ni técnica para gestionarlos. Sin embargo, las mujeres del hotel recibieron apoyo de instituciones gubernamentales y de educación superior que les han provisto de capacitación, tales como la Universidad Autónoma Metropolitana (UAM) campus Xochimilco, Universidad Iberoamericana de Puebla y México, la Secretaría de Turismo (SECTUR) y Secretaría de Agricultura, Ganadería, Desarrollo Rural, Pesca y Alimentación (SAGARPA). Los temas en los cuales se les capacitó fueron: administración de empresas, registro de marca, diseño de bordados, corte y confección de camisas, comercialización de productos, aspectos relacionados con el distintivo $\mathrm{M}$, derechos de la mujer, entre otros.

Referencias:

Alonso, D., González, N. y Nieto, M. "Emprendimiento social vs innovación social" Cuadernos Aragoneses de Economía, Vol. 24, No. 1-2, 2015, 119-140.

Deere, Carmen Diana y Magdalena León (2000). Género, propiedad y empoderamiento: tierra, Estado y mercado en América Latina, Bogotá, Colombia: Tercer mundo Editores en coedición con el Programa de Estudios de Género, Mujer y Desarrollo, Facultad de Ciencias Humanas, Universidad Nacional de Colombia.

Duarte, T., Ruiz Tibana, M. Emprendimiento, Una opción para el desarrollo" Revista Scientia Et Technica, Vol. XV, No. 43, 2009, 326-331. Fischel Volio, A. (2013). "Red Latinoamericana de Universidades por el emprendedurismo social" (en línea), 2013, consultada en internet el 28 de abirl de 2018. Dirección de internet: http://www.redunes.org/wp-content/uploads/2013/07/ Congreso-Emprendedurismo-Social-Ponencia-Astrid.pdf

Kliksberg, B. (2011). Emprendedores sociales. Los que hacen la diferencia. Temas Grupo Editorial. Lastarria-Cornhiel, S. (2008). Feminización de la agricultura en América Latina y África.

Tendencias y fuerzas impulsoras, RIMISP, Centro Latinoamericano para el Desarrollo Rural, Santiago de Chile. López Guerra, Verónica, Rojas, Olga Lorena "Rezagos en el nivel de autonomía de las mujeres rurales mexicanas en la primera década del siglo XXI" Revista de Estudios demográficos y urbanos (en línea), Vol. 32, No. 2, 315-354, 2017, consultada en internet el 7 de abril de 2018.

Dirección de internet: https://dx.doi.org/10.24201/edu.v32i2.1644

Martínez-Iglesias, M., Alarcón, A. A. (2013). Sociedades rurales, migración masculina y poder de negociación femenino.

Rural society, male migration, and female bargaining power (Working paper). Department of Sociology, Rovira i Virgili University, Catalonia, Spain.

Masehual (2016) Hilado nuestras historias. El camino recorrido hacia una vida digna, México.

Melián Navarro, A., Campos Climent, V. "Emprendedurismo y economía social como mecanismos de inserción sociolaboral en tiempos de crisis" REVESCO. Revista de Estudios Cooperativos, Norteamérica (en línea), Vol. 100, 2010. Consultado

el 20 de marzo de 2018. Dirección de internet: http://revistas.ucm.es/index.php/REVE/article/view/19552.

Palacios, G. "Emprendimiento social: integrando a los excluidos en el ámbito rural" Revista Scielo (en línea), Vol. 4, No.16, 2010. Consultado el

24 de marzo de 2018. Dirección de internet: $h t t p: / / w w w . s c i e l o . o r g . v e / s c i e l o . p h p ? s c r i p t=s c i \_a r t t e x t \& p i d=S 1315-95182010000400002$.

Rodríguez, A. y Flores, E. "Análisis comparativo entre empresas de emprendimiento social vs empresas tradicionales por medio de indicadores financieros clave" Revista Vinculatégica, No. 1, 2311-2338, 2016. Soler, P., \& Planas, A., \& Ciraso-Calí, A., \& Ribot-Horas, A. "Empoderamiento en la comunidad. El diseño de un sistema abierto de indicadores a partir de procesos de

Evaluación Participativa" Revista de Pedagogía Social Interuniversitaria, No. 24, 49-77, 2014.

Vázquez Luna, D., Mortera, D., Rodríguez, N., Martínez, M., Velázquez, M.G. (2013) “Organización comunitaria de mujeres:

del empoderamiento al éxito del desarrollo rural sustentable". Revista de Estudios de Género, la ventana (en linea) Vol. 5, 2013,

consultada el 30 de marzo de 2018. Dirección de internet: http://www.redalyc.org/articulo.oa?id=88428978011.

Vizcarra-Bordi, I., Lutz, B., \& Ramírez-Hernández, R. "El mismo fogón: migración y trabajo reproductivo femenino en comunidades mazahuas" Convergencia. Revista de Ciencias Sociales, Vol. 20, No. 61, 2013, 193-218. 